\title{
High yield fabrication of fluorescent nanodiamonds
}

\author{
Jean-Paul Boudou ${ }^{1,6}$, Patrick A Curmi ${ }^{1,6}$, Fedor Jelezko ${ }^{2}$, \\ Joerg Wrachtrup ${ }^{2}$, Pascal Aubert ${ }^{3}$, Mohamed Sennour ${ }^{4}$, \\ Gopalakrischnan Balasubramanian ${ }^{2}$, Rolf Reuter $^{2}$, Alain Thorel ${ }^{4}$ \\ and Eric Gaffet ${ }^{5}$ \\ ${ }^{1}$ Structure and Activity of Normal and Pathological Biomolecules-INSERM/UEVE U829, \\ Université d'Evry-Val d'Essonne, Bâtiment Maupertuis, Rue du père André Jarlan, F-91025 \\ Evry, France \\ 2 3.Physikalisches Institut, University of Stuttgart, Pfaffenwaldring 57, D-70550 Stuttgart, \\ Germany \\ ${ }^{3}$ Nanometric Media Laboratory, Université d'Evry-Val d' Essonne, Bâtiment Maupertuis, \\ Rue du père André Jarlan, F-91025 Evry, France \\ ${ }^{4}$ Centre des Matériaux, Mines Paris, ParisTech, BP 87, F-91000 Evry, France \\ ${ }^{5}$ Nanomaterials Research Group-UMR 5060, CNRS, UTBM, Site de Sevenans, \\ F-90010 Belfort, France
}

E-mail: jpb.cnrs@free.fr,pcurmi@univ-evry.fr and f.jelezko@physik.uni-stuttgart.de

\begin{abstract}
A new fabrication method to produce homogeneously fluorescent nanodiamonds with high yields is described. The powder obtained by high energy ball milling of fluorescent high pressure, high temperature diamond microcrystals was converted in a pure concentrated aqueous colloidal dispersion of highly crystalline ultrasmall nanoparticles with a mean size less than or equal to $10 \mathrm{~nm}$. The whole fabrication yield of colloidal quasi-spherical nanodiamonds was several orders of magnitude higher than those previously reported starting from microdiamonds. The results open up avenues for the industrial cost-effective production of fluorescent nanodiamonds with well-controlled properties.
\end{abstract}

\section{Introduction}

Diamond nanoparticles have received increasing attention owing to their outstanding prospective applications in various areas of physics, chemistry and biology [1-5]. Most of these applications are based on the unique optical and magnetic properties associated with defects in diamond. Among these, red/near-infrared fluorescent nanocrystalline diamonds are new nano-objects with several applications in biology (biomolecule labeling/vectorization, bioanalysis) [3], quantum information processing (quantum cryptography and quantum computing) $[6,7]$, magnetometry and novel imaging techniques [1]. The nitrogen-vacancy (NV) defect, responsible for the diamond red/near-infrared fluorescence, is a particular type of optically active defect (color center) that deserves special consideration [8]. First, the fluorescence of the NV

6 These authors contributed equally to this work. center in diamond is unconditionally stable, i.e. it does not photobleach or blink even under high excitation intensities. [9]. Second, NV-containing diamonds have the potential to be one of the smallest nanoparticle labels for use in biology, all other nanoparticle labels being limited to the range of tens of nanometers. For example, even though the smallest quantum dots themselves have a size of a few nanometers, encapsulation is needed for their chemical isolation from the cell for both optical stability and cell toxicity issues. Blinking also remains problematic when tracking of quantum dots is required. In the case of metal nanoparticles, light scattering scales as the inverse sixth power of the size and the detection limit is normally reached for a few tens of nanometers. In contrast, NV color centers remain stable when localized beyond 1-2 $\mathrm{nm}$ of the surface of bulk diamond [10], and so the potential exists to create NV-containing nanodiamonds of a few nanometer diameters. Third, aside from the toxicity issues of nanoparticles in general, diamond is known for 
its extreme chemical and biological inertness [11], even if nanotoxicology in general is still under investigation. The native diamond surface can be either hydrophilic, oxygenterminated, or hydrophobic, hydrogen-terminated [12]. This gives a very wide range of options for surface functionalization and grafting of biomolecules, such as proteins or DNA [13].

To date, a few methods exist to produce diamond nanoparticles. Ultradispersed detonation diamond nanoparticles can be obtained with a controllable size [14] and a very convenient $\mathrm{sp}^{2}$ shell [15] for functionalization and bioconjugation, but they do not contain any $\mathrm{C}$-centers (isolated substitutional nitrogen atoms in the diamond lattice), but impurities such as surface-or lattice-aggregated nitrogen and metals in significant amounts. More recently, dispersed nanodiamonds, just several nanometers in diameter, have been synthesized at only a laboratory scale from carbon black by laser irradiation in water at room temperature and normal pressure [16]. Finally, nanodiamonds have been made by using a microwave plasma torch technique with methane and Ar or $\mathrm{N}_{2}$ as catalysts [17]. The production of diamond nanocrystals containing NV centers can be achieved by the chemical vapor deposition technique (CVD) [18]: however, incorporation of nitrogen in small nanocrystals by this means is very inefficient [19]. Another option is to make NV centers by implanting nitrogen ions into pure diamond crystals $[10,20]$. Yet, the penetration depth of nitrogen ions is quite limited (approximately $1 \mu \mathrm{m} \mathrm{MeV}^{-1}$ ) and the production of large samples using this technique seems to be difficult. Similarly NV centers can be made in the nanodiamond itself by cold implantation with an in situ annealing procedure to incorporate homogeneously a large number of isolated nitrogen atoms on substitutional sites in an undamaged diamond environment over an area of a few square centimeters. The sample depth is limited to a few nanometers in this case.

Stable and bright fluorescent NV-rich nanodiamonds can be fabricated by irradiation and annealing in vacuum of type Ib high pressure high temperature (HPHT) diamonds grown from metal catalysts in a few minutes. In this synthetic diamond, nitrogen comes from the solvent metal and carbon source material, as well as from the residual gas left in the HPHT reactor. This nitrogen dissolves in the solvent metal and is incorporated in the diamond crystal during growth as substitutional nitrogen atoms remaining isolated for thermokinetics reasons. Such an $\mathrm{N}$ atom (called a C-center, single $\mathrm{N}$ or $\mathrm{N}_{\mathrm{S}}$ ) is bound to four other diamond lattice carbon atoms. The $\mathrm{C}$-defect center (approximately $>50 \mathrm{ppm}$ ) gives a yellow color due to optical absorption. During irradiation with photons, electrons, ions or neutrons, energetic particles knock diamond carbon atoms out of their normal lattice position by creating vacancies (V). Subsequent annealing (typically at $800^{?} \mathrm{C}$ under vacuum) leaves single $\mathrm{N}$ immobile but activates the migration of $\mathrm{V}$ towards them leading to the formation of fluorescent NV centers. Up to now, this HPHT route has been used as follows: a first mechanical milling step of as-grown HPHT diamond ( $>20-50 \mu \mathrm{m}$ ) yields a very small and expensive fraction of nanodiamonds [21]. Fluorescent nanoparticles are then produced by irradiation and annealing in vacuum of the nanodiamond fraction: however, just a part
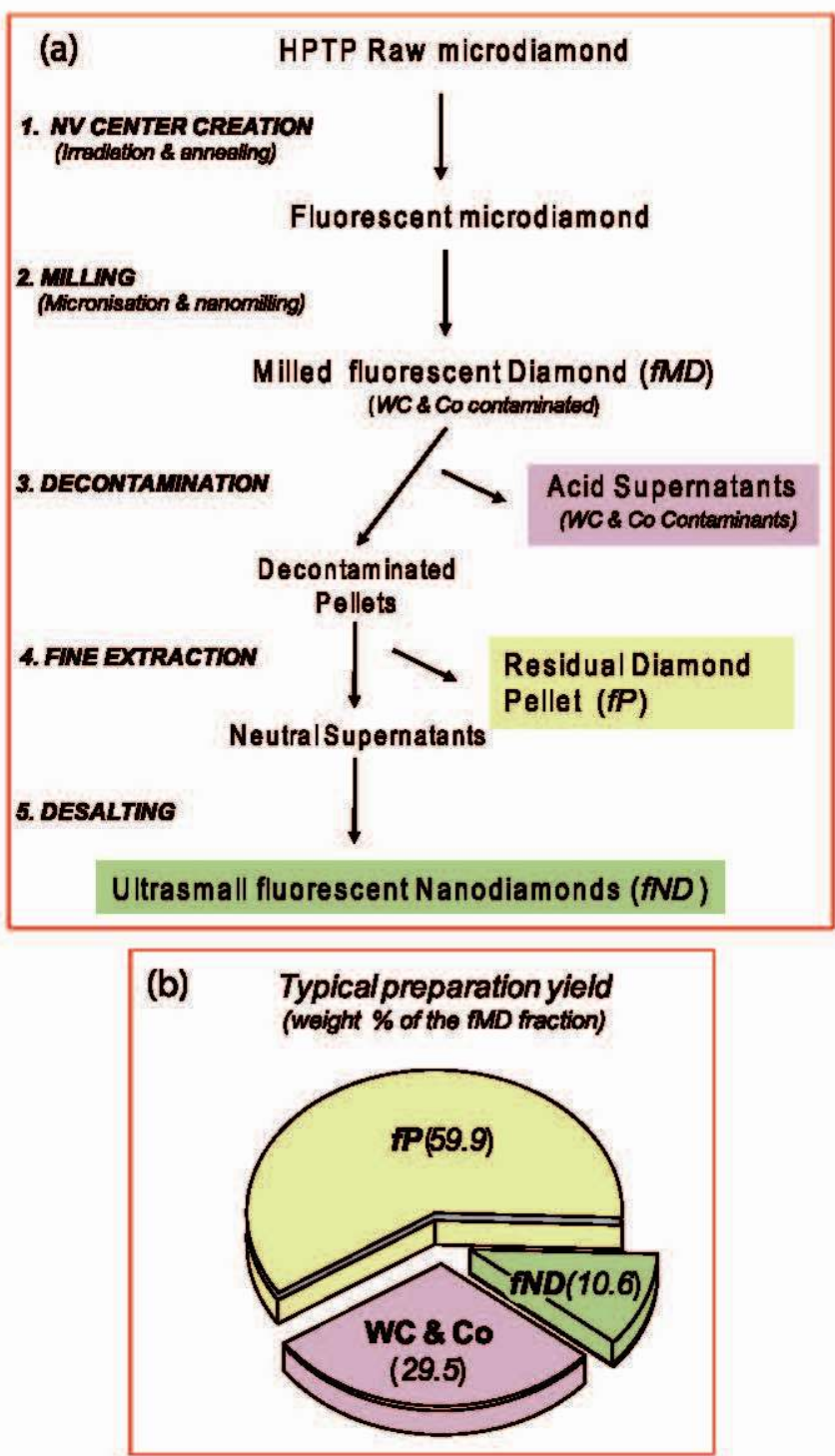

Figure 1. Flow chart for fluorescent ultrasmall nanodiamond (fND) preparation. (a) NV creation is obtained by $10 \mathrm{MeV}$ electron irradiation of raw HPHT microdiamond followed by annealing at $800^{?} \mathrm{C}$ under vacuum. Milling of the fluorescent microdiamond is performed in two steps, micronization and nanomilling. Decontamination includes a hot $\mathrm{HF} / \mathrm{HNO}_{3}$ attack followed by a series of centrifugation steps. The first pellet is resuspended in a small MQW volume. The second pellet is resuspended in a large MQW volume and $\mathrm{NH}_{4} \mathrm{OH}$ neutralized. Supernatants obtained by three subsequent centrifugations are pooled and desalted by ultrafiltration. (b) Mass distribution of the metal-contaminated diamond (fMD) fractions expressed in dry weight per cent. The WC + Co fraction mass was obtained by subtracting the pellet (fP) and fND mass from the fMD mass.

of this fraction can actually be rendered fluorescent due to still debated reasons [22-25].

We describe here for the first time an industrial scalable method [26] which allows the efficient conversion of asgrown HPHT diamond microcrystals into spatially isolated ultrasmall quasi-spherical NV-rich diamond nanoparticles showing stable non-bleachable fluorescence (figure 1). In the present state of its development, the overall yield of this 
method reaches about $15 \%(\mathrm{w} / \mathrm{w})$ of the microdiamond mass converted into $\leqslant 10 \mathrm{~nm}$ diamond nanoparticles. It opens realistic perspectives for the preparation of large samples of homogeneously fluorescent nanodiamonds necessary for research and development purposes.

\section{Experimental procedure}

\subsection{Raw material}

The raw initial material was a highly crystalline synthetic micron diamond powder (Element Six PDA999) containing 80-100 mesh (150-190 $\mu \mathrm{m})$ blocky, very uniformly cubooctahedral shaped diamond crystals with extremely high toughness, thermal stability and impact strength.

\subsection{Electron irradiation and annealing}

Electron irradiation was performed with a Rhodotron accelerator (Ionmed, Spain) operating at a beam power of $80 \mathrm{~kW}$ (10 MeV and $8 \mathrm{~mA}$ ) distributed over around $250 \mathrm{~cm}^{2}$ giving a power density of $320 \mathrm{~W} \mathrm{~cm}^{-2}$ (i.e. $7200 \mathrm{~W} \mathrm{~cm}^{-2} / 24 \mathrm{~h}$ ). The sample was placed in a 316 grade stainless steel $(900 \mathrm{~mm} \times$ $20 \mathrm{~mm} \times 5 \mathrm{~mm}$, wall thickness: $0.5 \mathrm{~mm}$ ) vessel cooled in fresh running water. The diamond layer to be penetrated was $\leqslant 4 \mathrm{~mm}$ over an area of $180 \mathrm{~cm}^{2}$.

After electron irradiation, the sample was annealed at $800^{?} \mathrm{C}$ under vacuum (? $10^{-8}$ Torr) during $2 \mathrm{~h}$ to allow migration of vacancies (V), created by electron irradiation. and NV color centers formed by the combination of $\mathrm{V}$ with isolated substitutional nitrogen atoms $\left(\mathrm{N}_{\mathrm{S}}\right)$ occurring in the crystal lattice of $\mathrm{Ib}$ type diamonds [27, 28].

\subsection{Milling}

To convert microdiamonds into smaller particles, we first used nitrogen jet milling autogeneous micronization which was preferred to planetary milling with metallic beads because the latter resulted in severe contamination by metals. A $250 \mathrm{~g}$ sample of raw initial material was first milled into a fine pure powder for $2 \mathrm{~h}$ in a $100 \mathrm{AFG}$ fluidized bed opposed jet mill (Hosokawa-Alpine, Germany) with a nitrogen flow rate of $60 \mathrm{~m}^{3} \mathrm{~h}^{-1}$ and a high grinding pressure ( $8 \mathrm{bar}$ ). After this step, we obtained a fine pure gray powder (with $97 \%$ of particles having sizes below $2 \mu \mathrm{m}$ ).

A nanomilling procedure was then used to convert the jet milling product into fluorescent nanodiamonds (fND). A ten gram aliquot was thus ball milled under argon using a planetary ball mill (Vario-Planetary Mill, Pulverisette 4, Fritsch, Germany) [29,30], with hard alloy WC+6\% Co bowls and lids equipped with two valves (for milling under argon) and $3010 \mathrm{~mm}$ balls made with the same WC-Co cemented carbide. The powder-to-ball weight ratio $R$ was $1 / 35$. The absolute speed of the main disc was $400 \mathrm{rpm}$ and the relative rotation speed of the vials with respect to the supporting disc was -2.17 .

The program extended for $72 \mathrm{~h}$ as follows: the aliquot was ball milled for successive periods of $15 \mathrm{~min}$, each separated by a $30 \mathrm{~min}$ cooling period ( $24 \mathrm{~h}$ effective grinding time).
The temperature measured on the outside bowl wall was about $50^{\text {? }} \mathrm{C}$. This programed stepwise grinding mode was found to be more convenient than flowing liquid nitrogen around the vial to control the milling temperature between room temperature and $50^{?} \mathrm{C}$.

\subsection{Purification and colloidal dispersion}

After milling, the beads were recovered by sieving the fluorescent milled diamond ( $\mathrm{FMD}$ ). The fine tungsten carbide particles produced by milling present in the fMD sample, and not recovered by sieving, were dissolved by harsh acid treatment: a $750 \mathrm{mg}$ aliquot of the fMD sample was placed in a $100 \mathrm{ml}$ Teflon autoclave ( $200 \mathrm{ml}$ Zeoclave-Autoclave France) with $30 \mathrm{ml}$ of a hydrofluoric and nitric acid mixture $(2: 1 \mathrm{v} / \mathrm{v})$ at $150^{?} \mathrm{C}$ for $48 \mathrm{~h}$.

After completion of the acid treatment, an excess of Milli-Q ultrapure water (MQW), up to $100 \mathrm{ml}$, was added to the sample which was then mechanically dispersed before centrifugation $(4000 \mathrm{~g}, 30 \mathrm{~min})$. The diamond sample precipitates under these acid conditions, which allows easy recovery from the acid. To refine fND purification, this first pellet was suspended by strong shaking in a small MQW volume to keep a strongly acid suspension. After centrifugation $(4000 \mathrm{~g}, 30 \mathrm{~min})$ the strongly acid supernatant was discarded and the pellet which contained precipitated nanodiamonds was then suspended in $50 \mathrm{ml}$ of MQW. This new suspension was finally exactly neutralized with aqueous ammonia solution and centrifuged again $(4000 \mathrm{~g}, 30 \mathrm{~min})$. The resulting supernatant containing fine diamond nanoparticles was stored for further processing. The residual pellet was resuspended (in $50 \mathrm{ml}$ of $\mathrm{MQW}$ ) and centrifuged (4000g, $30 \mathrm{~min}$ ) twice more to complete the extraction of the diamond nanoparticles. The neutral supernatants were then pooled and desalted by tangential flow filtration using a Millipore Pellicon $\mathrm{XI}$ cassette equipped with a Biomax membrane. The purified samples obtained were called the residual fluorescent pellet (fP) and fluorescent nanodiamond fND (pooled, concentrated and desalted supernatants). fP and fND samples were dried using a Rotavapor. An aliquot was taken for x-ray diffraction and surface group analysis. Another one was resuspended in MQW for transmission electron microscopy observations and EDX analysis. The fP and fND masses were expressed either relative to the fMD mass or the total mass of the dried pure diamond (fP + fND).

\subsection{Transmission electron microscopy}

TEM analyses were performed on an FEI F-20ST (Philips) field emission gun transmission microscope equipped with super-twin polar pieces and operated at $200 \mathrm{kV}$. Energydispersive X-ray (EDX) analysis coupled with TEM was used to identify the elemental composition of selected areas. Images were recorded at approximately Scherzer defocus on a CCD multiscan camera after astigmatism corrections, and eventually filtered via the Digital Micrograph software. Materials for transmission electron microscopy, prepared by ultrasonic dispersion of the samples in water for $5 \mathrm{~min}$, were deposited on a copper grid coated with holey carbon. The deposited 
suspensions were thereafter dried in air prior to transmission electron microscopy analyses. To avoid any electron irradiation damage that could induce allotropic transformations of carbon materials, we used a reduced beam intensity but we did not observe any phase change, even after long exposure, as observed for detonation nanodiamond [15]. X-ray diffraction (XRD) was used to determine the sample structure and composition after milling, and after milling and purification. The data were collected using an X-ray diffractometer with $\mathrm{Cu} \mathrm{Ka}(\lambda=1.54056 \AA)$ radiation.

\subsection{Surface group analysis}

Analysis of the surface groups was done by temperatureprogramed desorption mass spectrometry. A small dried sample of about $5 \mathrm{mg}$ of diamond nanocrystals was placed in a crucible and heated from 100 to $1450^{?} \mathrm{C}$ at $30^{?} \mathrm{C} \mathrm{min}^{-1}$ at atmospheric pressure in helium with one volume per cent of neon at a flow rate of $10 \mathrm{~cm}^{3} \mathrm{~min}^{-1}$. Evolved gases were continuously monitored and quantified with a mass spectrometer. The $\mathrm{CO}$ evolution profile was corrected from the $\mathrm{m} / \mathrm{z} 28$ contribution due to molecular nitrogen. The system was calibrated using gas mixtures in pure He with $1 \%$ Ne. The signal amplitude of each mass was compared to the $m / z 20$ signal amplitude of Ne. Physically desorbing water from heated samples produced a broad peak, probably due to interactions between polar water molecules and the inner walls of the transfer line between oven and detector. The integrated water peak was used to assess sample moisture content, and hence to correct the gas yield with respect to organic carbon or total carbon content on a dry, ash-free basis.

\section{Results and discussion}

\subsection{Nanoparticle characterization}

XRD analyses of the $\mathrm{IMD}$ sample show, together with diamond, the presence of WC nanoparticle contaminant originating from the milling vial and beads (figure 2(a)). However, the spectrum demonstrates that the diamond structure was not altered by the milling procedure. Indeed, no broad (002) peak corresponding to $\mathrm{sp}^{2}$-bonded carbon was observed, but only the c-diamond and WC ones. After WC removal, x-ray diffraction pattern performed on fND shows prominent peaks at angles corresponding to the normal structure of diamond, i.e. the (111), (220) and (311) reflections. Though it is known that high energy milling produces a high density of crystal dislocations [31], precise analyses processing of our XRD data did not show any significant change of the diamond lattice parameter. The presence of wide XRD diffraction reflection is due to both microstrain isolated substitutional nitrogen [32] and the small size of the particles which are stated to be in the range of several nanometers: the width of the peak is inversely related to the nanoparticle dimensions and can be approximated by the Scherrer equation [33]. The mean fND particle size estimated by this equation applied to the Gaussian-Lorentzian, Pearson VII or Voigt fitting curve of the (311), (220) or (111) diffraction peak is less than or equal to $10 \mathrm{~nm}$, whatever the crystallographic direction.

Energy-dispersive $\mathrm{x}$-ray (EDX) spectra show that the combined $\mathrm{HF} / \mathrm{HNO}_{3}$ treatment at $150^{\text {? }} \mathrm{C}$ for $48 \mathrm{~h}$ efficiently removes the contaminants from the fMD sample (figure 2(c)). EDX analyses also reveal considerable amounts of oxygen (mean value: $1.3 \mathrm{wt} \%$ ) coming from secondary oxygen chemisorption forming surface carbon-oxygen complexes [34] as also revealed and characterized by temperature-programed desorption (figure 2(d)). Carbon-oxygen surface complexes are co-desorbed with NO-containing surface groups (resulting from nitration during the acid treatment) and with $\mathrm{H}$-containing surface groups $(\mathrm{CH}, \mathrm{OH})$. The absence of fluorine in the desorbed gases indicates that there is no aqueous acid oxidative fluorination of the particles during the $\mathrm{HF} / \mathrm{HNO}_{3}$ treatment. This illustrates the fact that fluoride is a very poor nucleophile in aqueous solution and cannot cause surface oxygen group removal-as previously observed at higher temperature [35].

Assuming that the surface coverage ratios of the functional groups and diamond density remain unchanged, the differences observed between the thermal desorption profiles of the fND and the fP samples can be simply interpreted in terms of variation of specific surface areas. Since the sphere specific surface area is $3 / r \rho$ (where $r$ is the sphere radius and $\rho$ is the density), the total surface group density $\left(4.75 \mathrm{mmol} \mathrm{g}^{-1}\right.$ in fND and $1.45 \mathrm{mmol} \mathrm{g}^{-1}$ in P) can be inversely related to the mean particle size and hence the specific surface area. As a matter of fact, the fND/fP surface group density ratio (3.27) is of the same order of magnitude as the fP/fND mean particle size ratio deduced from HRTEM image processing (3.94). The higher $\mathrm{C}$ and $\mathrm{O}$ surface group density of nanodiamonds in the fND fraction, which represents about $17 \%$ of the purified diamond fraction ( $\mathrm{fND}+\mathrm{fP}$ ) after a milling time of only $24 \mathrm{~h}$ (figure 1(b)), would explain the good suspension stability of this fraction in pure neutral water. Surface modifications assisted by sonication are currently investigated to further improve the dispersion of both fractions in water over wide $\mathrm{pH}$ and ionic strength ranges.

The fully purified fND sample, obtained after decontamination and fine extraction, is made of pure nanocrystalline diamonds as shown in figure 3. HRTEM fast Fourier transform (FFT) phase images corresponding to the (111) lattice fringes did not show any local distortion of the diamond lattice. Nanodiamonds appear as deformed polygons with a quasispherical envelope and an aspect ratio varying from 1.13 to 1.75 (mean value $=1.37$ for the $2-50 \mathrm{~nm}$ particle size range) . This apparent quasi-spherical shadow with changing aspect ratios is best investigated further using electron tomography. Interestingly, the rounded shape of these particles, which derives from the initial uniformly cubo-octahedral-shaped diamond crystals, is noticeably different from the shape of commercial diamond nanoparticles obtained by steel ball milling of Ib type HPHT inclusion-rich micron diamond crystals selected for their friability (figure 4 ). The distribution of the diameters $d$ (the minor axis of the ellipse, taken instead of the diameter of a sphere of equivalent cross-sectional area on the image: $d=2(A / p)^{1 / 2}$, where $A$ is the projected area of the nanoparticle) shows excess skewness and kurtosis so that 

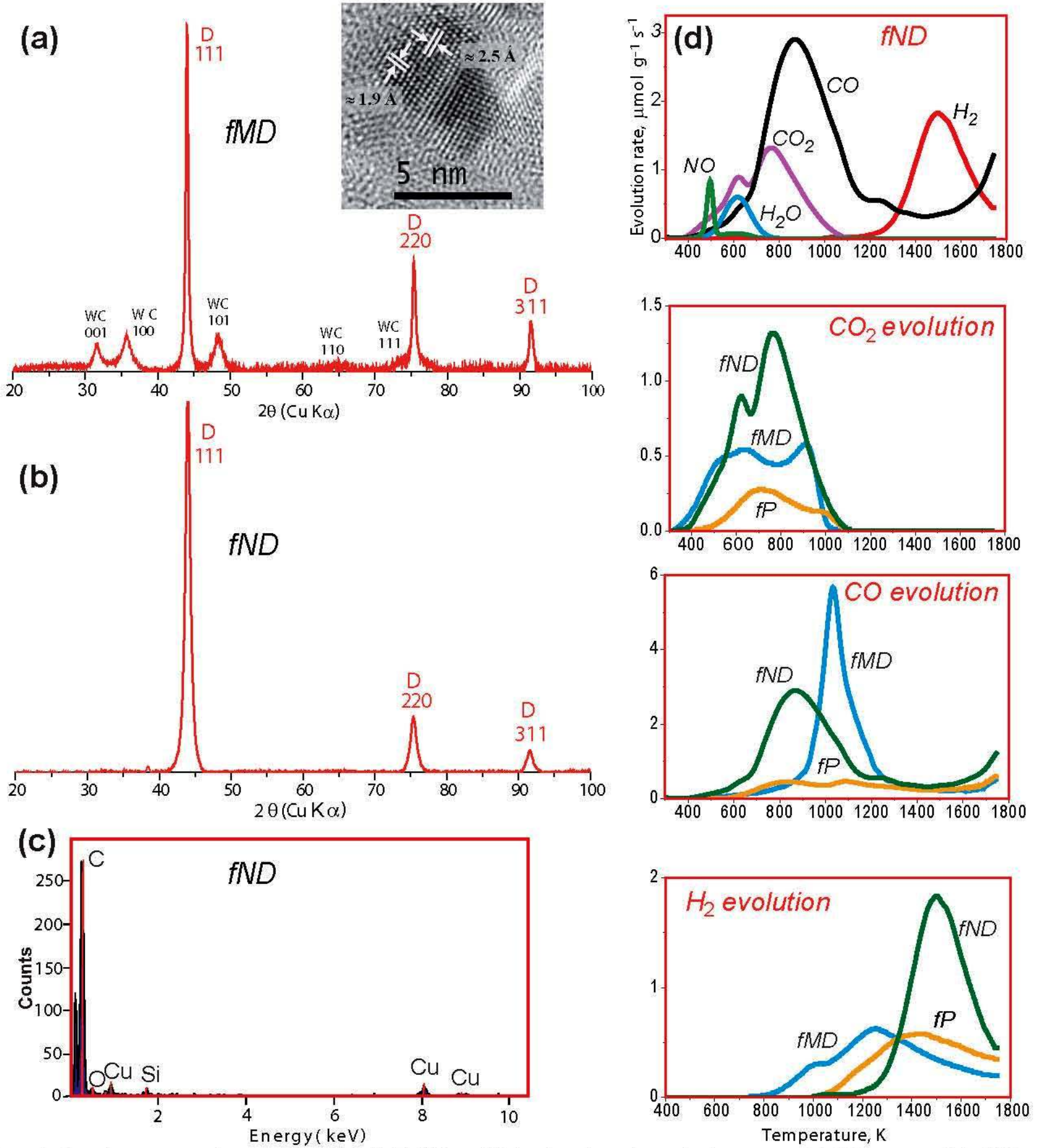

Figure 2. Sample structure and composition of the fMD, fND and $\mathrm{fP}$ fractions from the production process. (a) XRD pattern of the fMD sample showing a severe contamination with hexagonal WC. The WC diffraction peaks $\left(31.48^{\circ},(001), 35.76^{\circ}(100), 48.40^{\circ}(101), 64.04^{\circ}\right.$ $(110), 65.32^{\circ}(002), 73.20^{\circ}(111), 75.60^{\circ}(200), 76.92^{\circ}(102) 84.16^{\circ}(201)$, according to JCPDS $\left.25-1047\right)$ are indicated with $(h k l)$ values. The inset is the HRTEM image of a WC nanocrystal. Arrows show lattice fringes of the WC $\{100\}$ and $\{101\}$ planes. (b) XRD pattern of the fND sample showing intense peaks indexed to the cubic diamond structure $\left(43.97^{\circ}(111), 75.37^{\circ}(220), 91.59^{\circ}\right.$ (311); peak position and relative integrated intensities of each peak are within one standard deviation of the values given in JCPDS card no. 6-0675). (c) Energy-dispersive $\mathrm{x}$-ray (EDX) spectrum of the purified nanodiamond ( $\mathrm{fND}$ ) samples. The spectrum shows that the tungsten has been efficiently removed. The carbon $\mathrm{K} \alpha$ line remains as the only relevant peak in the spectrum, reflecting the purity of the diamond crystals. (d) Thermal desorption profiles of $\mathrm{CO}_{2}, \mathrm{CO}$ and $\mathrm{H}_{2}$ for the $\mathrm{fND}$ fraction and of either $\mathrm{CO}_{2}, \mathrm{CO}$ or $\mathrm{H}_{2}$ for the $\mathrm{fMD}$, fND and the centrifugation precipitate (fP) fractions.

they can be better fitted with the equation for the standard lognormal fND distribution with a mode occurring at 3.25 and a mean value of $3.5 \pm 0.3$ (figure 5). The lognormal character of the distribution probably results from the fact that, for a given magnification and set of instrument parameters, there is a resolution limit below which no size information is detectable. The mean sizes that we obtained by XRD and HRTEM, much below the minimum size $(30 \mathrm{~nm})$ predicted for 

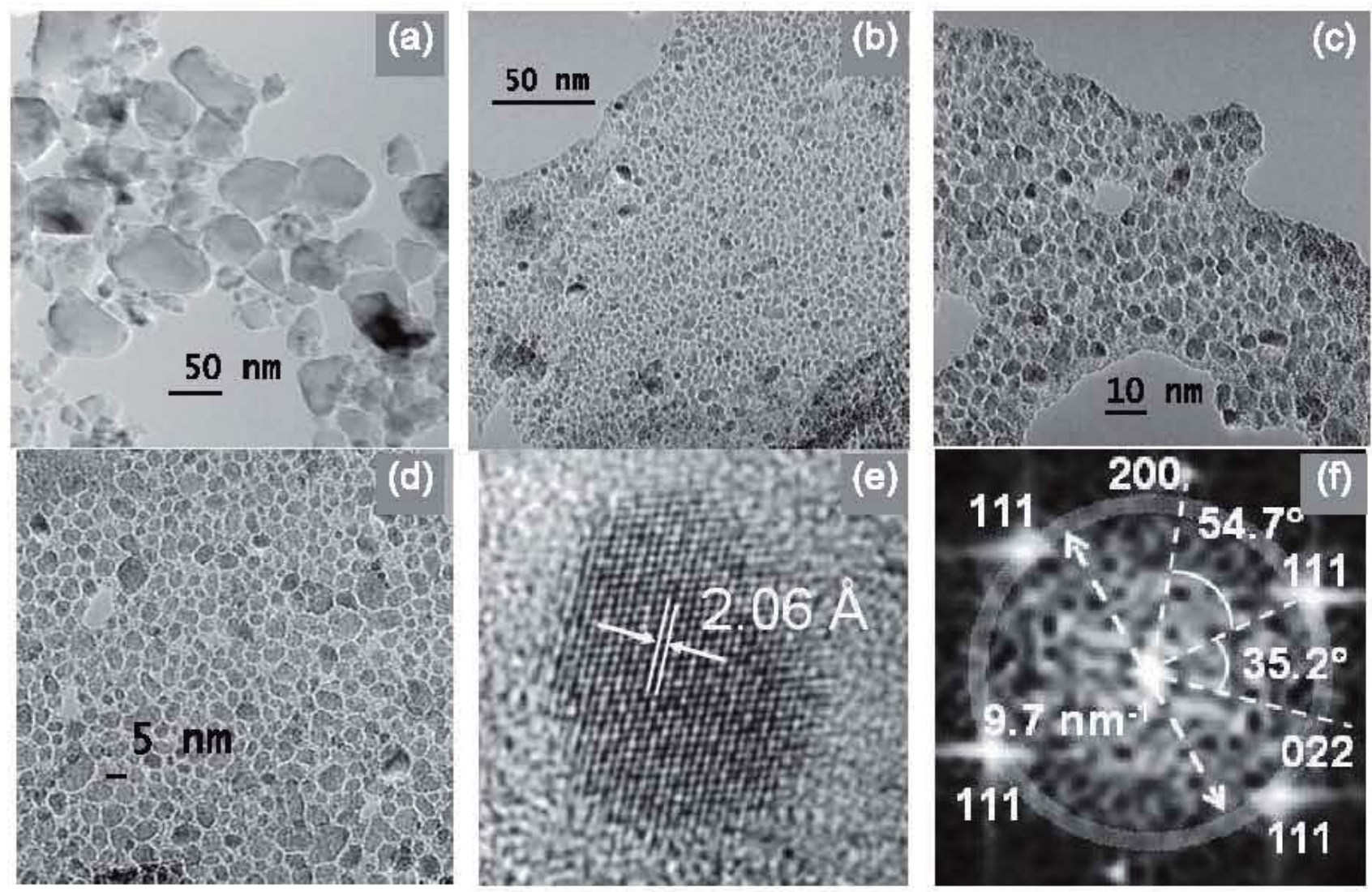

Figure 3. The milling method provides a homogeneous population of ultrasmall quasi-spherical diamond nanoparticles. TEM/HRTEM images from fP (a) and fND (b)-(d) as defined in figure 1. (e) HRTEM image of typical nanodiamond (f) FFT analysis of the nanodiamond crystalline structure where the $c$-diamond $d 111$ spacing is given by the inverse of the ring radius. The distance between the $\{111\}$ planes is equal to $a \sin \theta \approx 2.06 \AA$, where $a=3.5625 \AA$ is the unit cell dimension and $\theta=0.6154$ rad is the angle between the $\{111\}$ and the $\{110\}$ planes.

diamond grit particles produced by fracture [36], are of the same order of magnitude as those reported for the smallest synthetic or natural nanodiamonds-close to their predicted physicochemical stabilitylimit $[37,38]$.

\subsection{Fracture and amorphization during milling}

Our results suggest that, during both milling stages, HPHT diamond is simply broken without any amorphization, as can be observed for other materials [39-41]. Crystal breaking occurs with brittle fracture along the preferred octahedral \{111\} planes-a mechanism originally put forward by Tolkowsky [42]. The dominance of \{111\} cleavage in the crystallographic fracture of diamond is explained by the markedly lower strength and energy for fracture al ong these planes. This is inferred from first-principles calculations and also qualitatively explained by strain accommodation and the introduction of powerful 'bond-bending' energy terms upon stretching the crystal away from the \{111\} direction [43]. Yet, the mechanical properties of nanodiamond are still not well understood. From the calculated elastic constants and the estimated hardness, it has recently be en observed that nanodiamond softens as the size of its grains decreases [44]. The observed sof tening would be attributed to the enhancedfraction of interfacial atoms as the average grain size becomes smaller.
The production of ultrasmall quasi-spherical nanodiamonds from HPHT diamond grit described in this paper is spectacular and has never been reported before with standard mills [21]. Reasons for such results could be explaine $d$ by the specific performance of the vario-planetary mill (Pulverisette 4) $[30,45]$ for nanomilling of ultrahard and brittle materials. During planetary ball mills, due to the overlapping of grinding bows and supporting disc, the material to be ground and the grinding balls execute movements and trajectories in the grinding bow, which are defined by the transmission ratio. With standard planetary ball mills the grinding bow rotates at a fixed transmission ratio while with the vario-planetary mill the rotational speeds of grinding bows and supporting disc can be adjusted completely independently of each other, so that by varying the transmission ratio intermediate levels and combinations of frictional and impact pressures can be set as required. Ball milling process peak temperature and pressure, which can loc ally and instantly reach $300^{\circ} \mathrm{C}[46]$ and $12 \mathrm{GPa}$ [47], were not measured during our vario-planetary milling experiment but one can infer from our data that the $p, T$ parameters were below those for phase changes (amorphization, carbonization, burning) taking place during high pressure indentation [48], shear stress [49] or diamond polishing [50]. Our data suggest that the local temperature rise due to sliding/friction during diamond milling under argon 

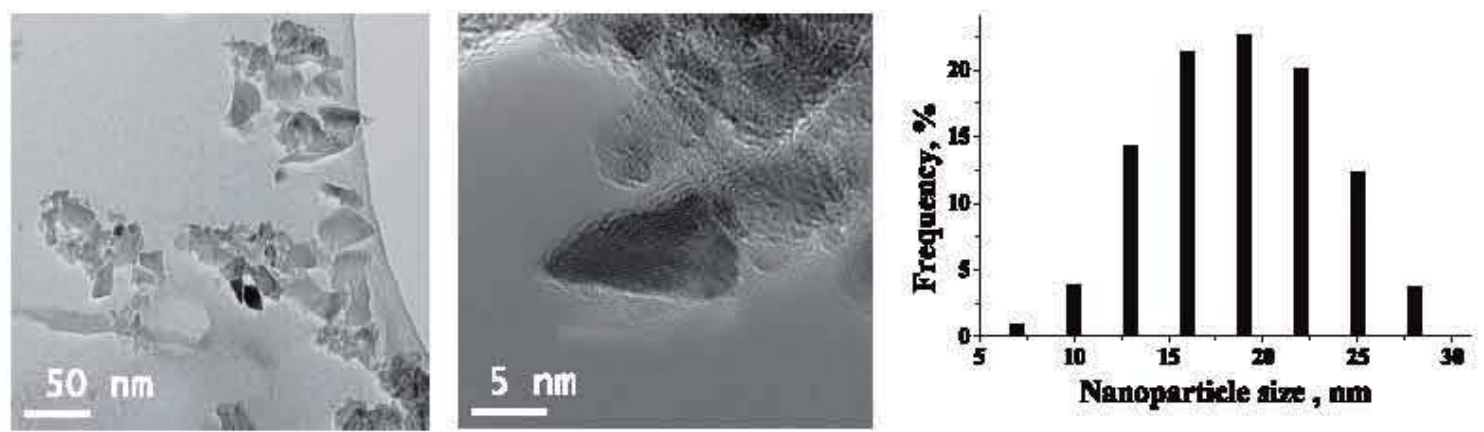

Figure 4. TEM/HRTEM images and particle size distribution of typical commercial nanodiamonds obtained by milling of HPHT di amond grit.
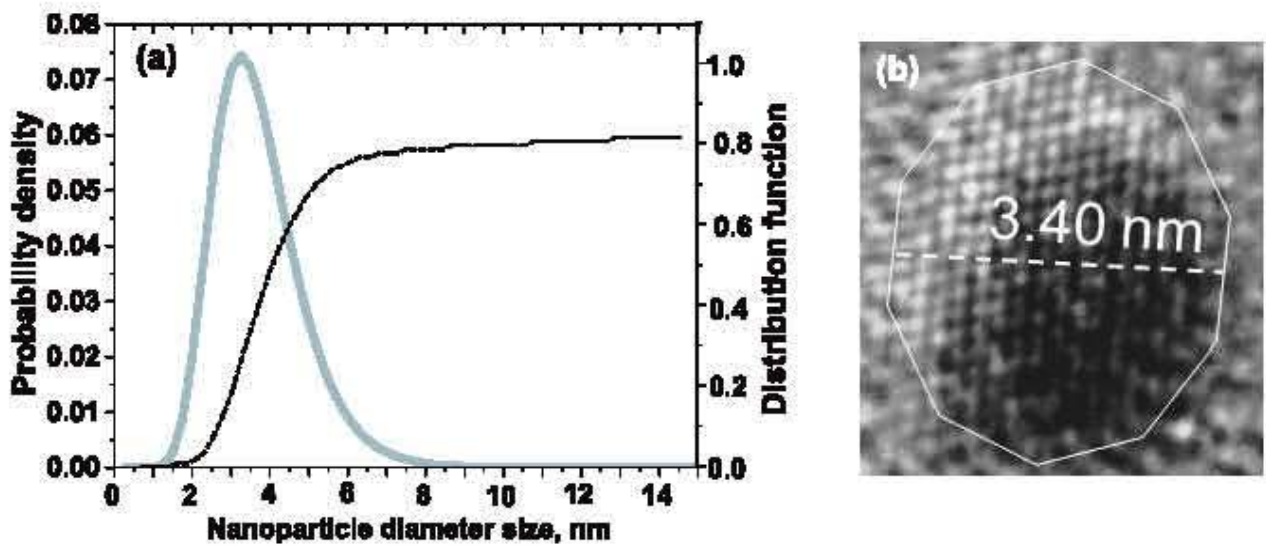

Figure 5. (a) Lognormal distribution of nanodiamond sizes from HRTEM images. (b) Example of polygonal slightly flattened contour placed over an HRTEM ellipsoidal nan odiamond image with its minor axis (broken line) taken as an approximation of the nanocrystal diameter. This measurement is calibrated both by the sc ale of observation and with the $\{111\}$ interplan ar spacing.

remained low enough to avoid any thermo(chemical) diamond alteration and enabling simple crystallographic fracture into nanodiamonds. For those small particles friction coefficient and specific wear rates would decrease with decreasing particle size [51] so that the smaller the particles become the more difficult it gets to reduce their size.

\subsection{Fluorescence properties of diamond nanoparticles}

One of the emerging applications of diamond nanocrystals is their use as fluorescent markers in microscopy. Such diamond luminescent labels are reported to be superior to conven tional chromophores owing to their unprecedented photostability.

In typical type Ib HPHT synthetic diamond, nitrogen atoms are homogeneously distributed if we consider diamonds with diameters higher than a few nanometers. On the other hand, nitrogen may be unevenly distributed at the level of the crystal unit cell (side $\approx 0.35 \mathrm{~nm}$ ) and may differ by a factor of two between the $\{100\}$ and $\{111\}$ growth sectors. In addition, there may also be some minor growth sectors, such as $\{113\}$ or $\{115\}$, where the nitrogen concentration is negligible [52]. For these ressons, the initial mass of isolated substitutional nitrogen atoms $\left(\mathrm{N}_{\mathrm{S}}\right.$, at a concentration of around $200 \mathrm{ppm}$ in the raw microdiamond) is equally divided by milling in individual nanocrystals (which each includes a large number of crystal unit cells). The resulting nanodiamonds should have an average concentration of $1-3 \mathrm{~N}_{\mathrm{S}}$ per $5 \mathrm{~nm}$ nanocrystal - potentially available for the formation of single nitrogen-vacancy (NV) color defects. However, during the fabrication process some initial $\left(\mathrm{N}_{\mathrm{S}}\right)$ are removed. $A$ minor fraction of these nitrogen atoms (estimated by the ratio between the number of electrons dispatched per volume unit and the number of atoms in the same volume) can be knocked out of their substitutional position by electron irradiation. One can assume that there is a negligible loss of single nitrogen atoms due to aggregation of single nitrogen to $A$ aggregates during irradiation-annealing-milling (unlikely at this nitrogen concentration and $p, T$ conditions) $[53,54]$. Another second cause of nitrogen loss is due to mechanical exhumation from the diamond core to $<10 \mathrm{~nm}$ nanoparticle surface during milling. This loss can be assessed by taking into account the strong increase, below a particle size of $10 \mathrm{~nm}$, of the edgelocated nitrogen atoms with respect to the total nitrogen atoms. $\left(\mathrm{N}_{\mathrm{B}} / \mathrm{N}\right.$ ) ratio (figure $6(\mathrm{a})$ ) and the decrease of the correlated quatemary carbon atoms/lattice carbon atoms' $\left(\mathrm{C}_{\mathrm{Q}} / \mathrm{C}_{\mathrm{L}}\right)$ ratio (figure $6(\mathrm{~b})$ ).

An increase of the final NV density in nanodiamonds, and consequently a reduction of the predictable large statistical fluctuations of the NV concentration in ultrasmall nanocrystals, could be achieved, either by nanomilling of fuorescent microdiamonds prepared with $\mathrm{N}_{\mathrm{S}}$-rich diamonds (e.g. $\mathrm{N}_{\mathrm{S}}=2000 \mathrm{ppm}$, as shown in figure $6(\mathrm{a})$ ), such 

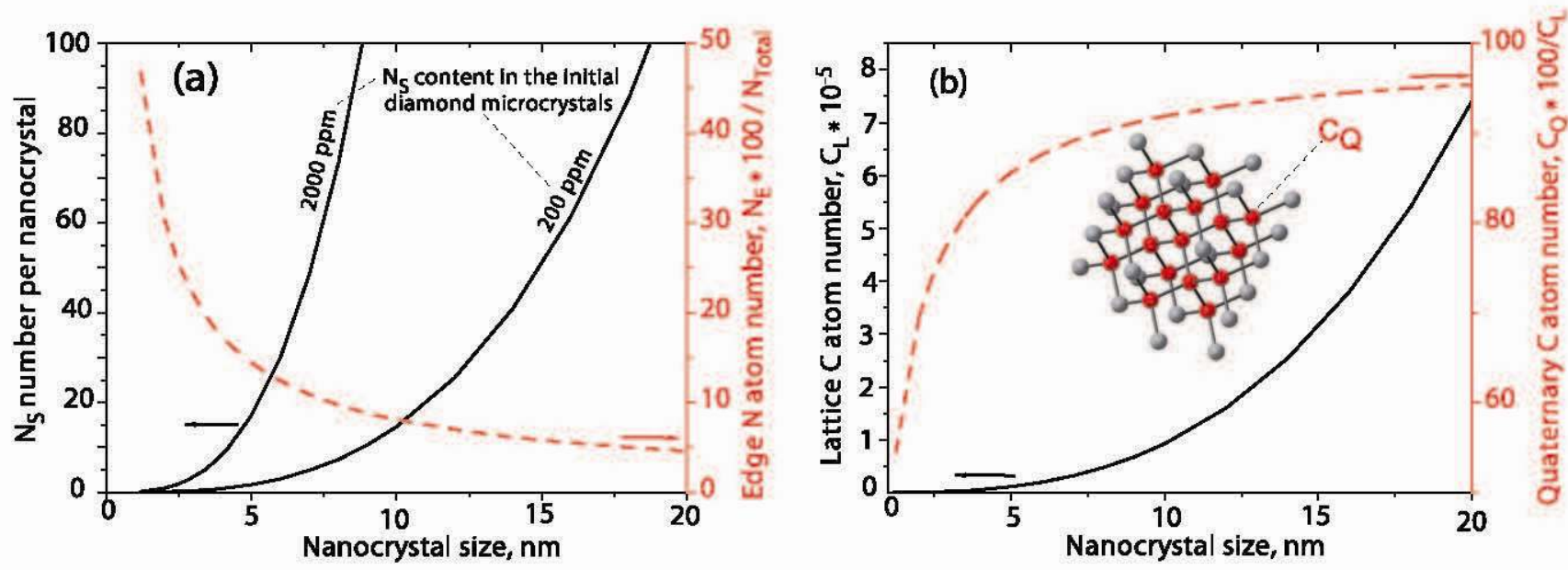

Figure 6. Predictable number of nitrogen (a) and carbon (b) atoms per typical diamond nanocrystal-assuming a negligible decrease of the theoretical density due to the decreasing fraction of quaternary atoms [44] and to the dislocations and lattice expansion (on the basis of XRD and HRTEM) possibly due to milling. (a) The $\mathrm{N}_{\mathrm{E}} / \mathrm{N}_{\text {Total }}$ ratio gives the fraction of nitrogen atoms exposed to the surface. (b) The $\mathrm{C}_{\mathrm{Q}} / \mathrm{C}_{\mathrm{L}}$ ratio gives the fraction of quaternary carbon atoms (as shown in the model inset) with respect to the total number of lattice carbon atoms $\left(\mathrm{C}_{\mathrm{L}}\right)$.

(a)

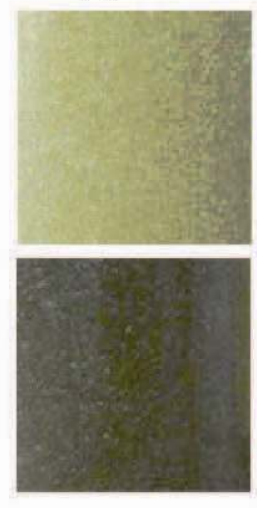

(b)

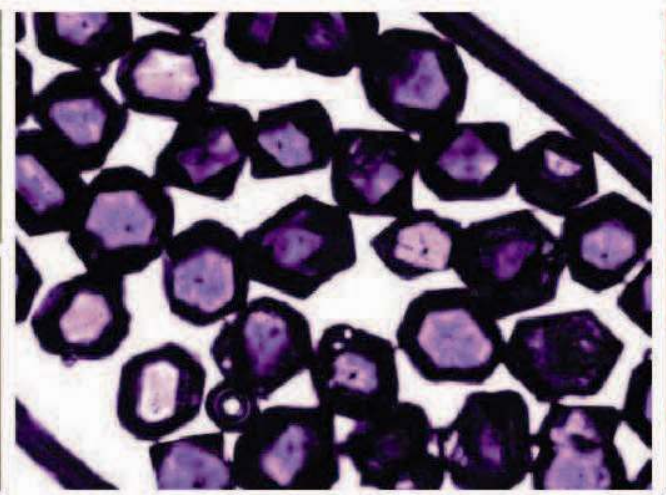

(c)

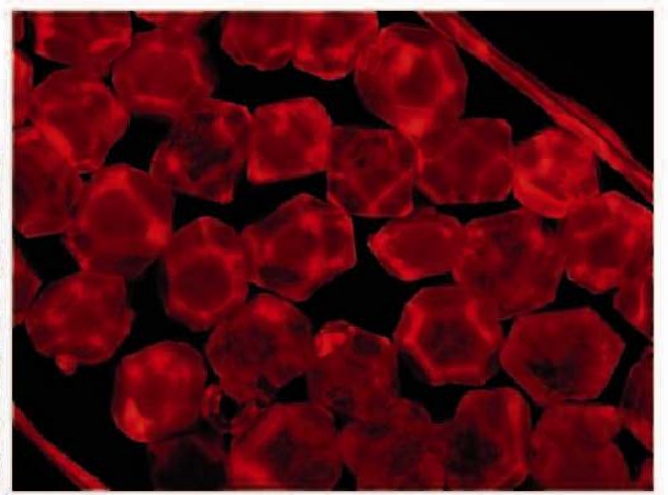

Figure 7. Optical observation of the raw 80-100 mesh HPHT diamond. (a) HPHT microdiamond seen with the naked eye: initial (above) and after electron irradiation (below). (b) Bright-field transmission image of the microdiamond after electron irradiation and annealing. (c) Image of the microdiamond after electron irradiation and annealing recorded with a fluorescence microscope under green excitation (20 ms duration).

as those recently synthesized with the $\mathrm{BN}-\mathrm{C}[55], \mathrm{Fe}_{3} \mathrm{~N}-$ $\mathrm{C}$ [56] or the $\mathrm{Ba}\left(\mathrm{N}_{3}\right)_{2}-\mathrm{C}$ systems [57], or by irradiationannealing of $\mathrm{N}_{\mathrm{S}}$-rich nanodiamonds produced by milling of these nitrogen-enriched microdiamonds-assuming that isolated substitutional nitrogen $\left(\mathrm{N}_{\mathrm{S}}\right)$ is stable in very small nanodiamonds [58] and does not aggregate in too large a proportion during vacancy creation and annealing [53, 54].

In our experiments diamond nanoparticles were prepared by electron irradiation $\left(10 \mathrm{MeV}, 5 \times 10^{19}\right.$ electrons $\left.\mathrm{cm}^{-2}\right)$ of type $\mathrm{Ib}$ HPHT micron diamond powder with a typical $\mathrm{N}_{\mathrm{S}}$ concentration of around $200 \mathrm{ppm}$ (Element Six PDA999) containing $8-100$ mesh $(150-190 \mu \mathrm{m})$. The sample was annealed under vacuum (? $10^{-8}$ Torr, $800^{?} \mathrm{C}$ during $2 \mathrm{~h}$ ), before milling and subsequent purification (figure 7). The resulting nanodiamonds were investigated using atomic force microscopy combined with a home-made optical microscope. Figure 8 shows a topography image of nanocrystals in $\mathrm{fP}+\mathrm{fND}$ fractions obtained by milling of the fluorescent microdiamond and purification of the fMD fraction (prepared by electron irradiation and annealing of type Ib HPHT with an $\mathrm{N}_{\mathrm{S}}$ concentration of around $200 \mathrm{ppm}$ ) immobilized on the glass surface. In order to examine relationships between the size of nanocrystals and their fluorescence, we recorded confocal images of the same sample area (marked with a square frame in figures 8(a) and (b)). Remarkably all nanocrystals present in the AFM picture show stable non-bleachable fluorescence. The brightness of individual spots differs significantly owing to different volumes of nanocrystals present in the sample. This distinguishes fluorescent nanocrystals from quantum dots where a change of size shifts the spectrum without affecting the oscillator strength of the optical transition. The fluorescence spectrum presented in figure $8(\mathrm{~d})$ shows the characteristic shape of the negatively charged NV defect. Note that the $637 \mathrm{~nm}$ zero-phonon line characteristic for NV color centers in bulk diamond is absent in nanocrystal spectra, indicating strong strain [59]. Such strain leads to splitting of the excited state causing inhomogeneous broadening of the zero-phonon line for nanocrystals containing many defects [60]. 


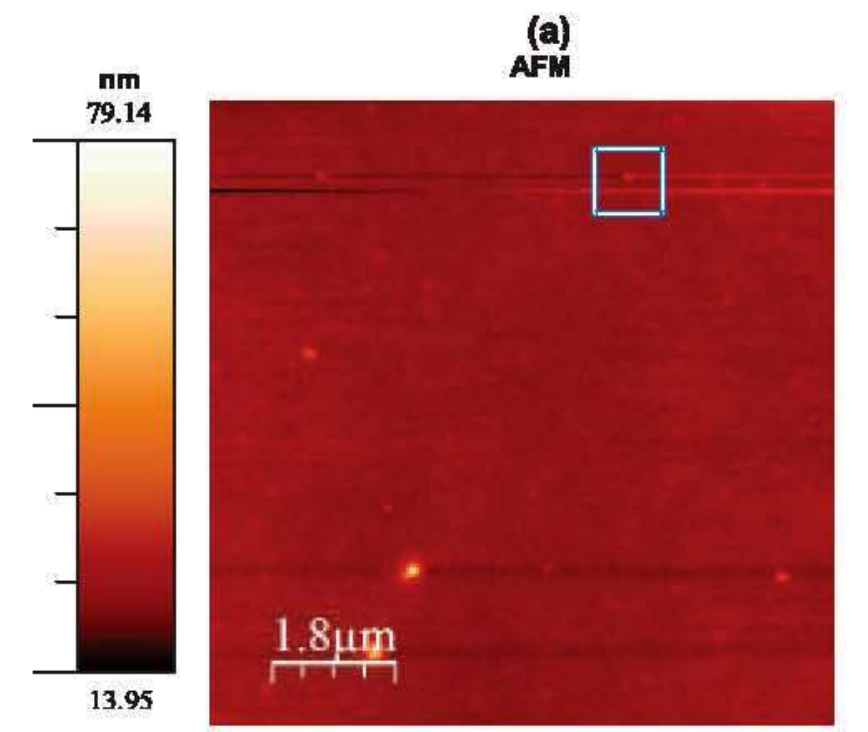

(c)

(a)

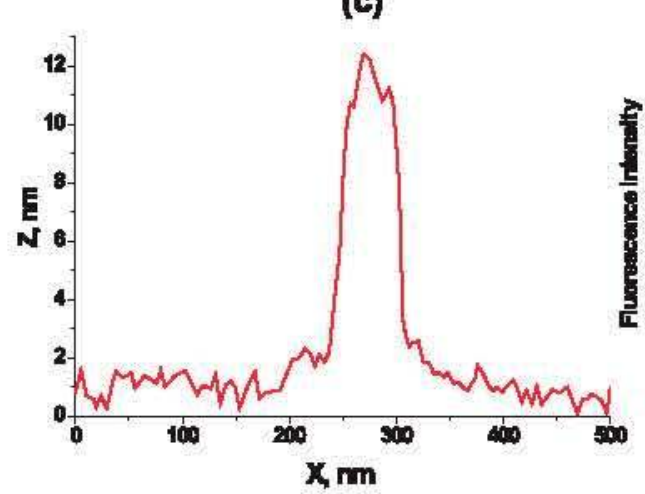

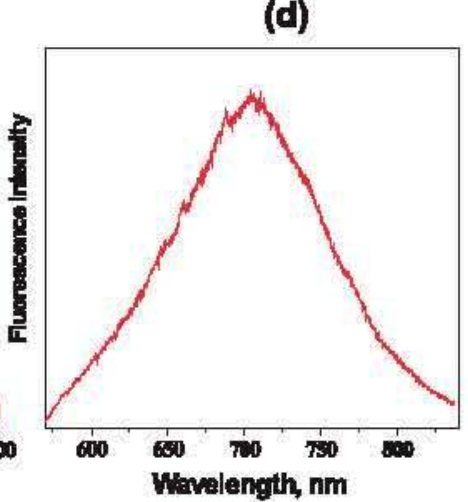

(b)

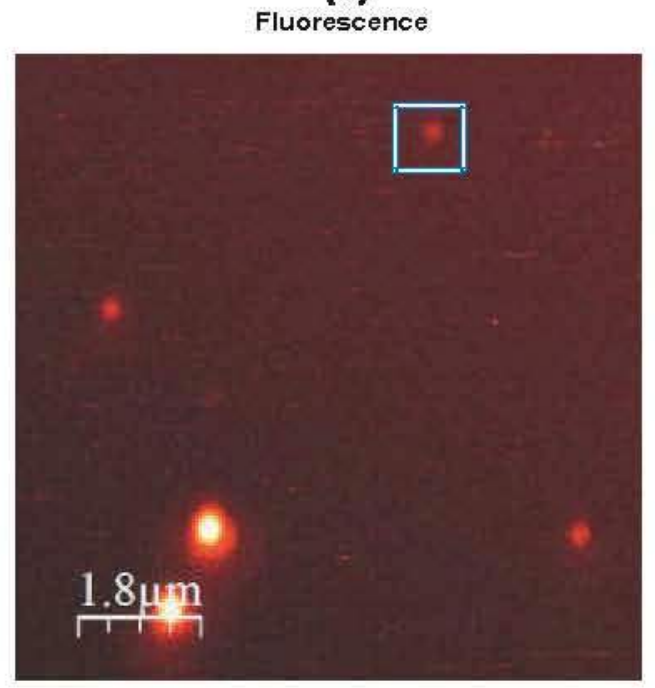

(e)

Figure 8. Atomic force microscopy (a) and photoluminescence microscopy (b) images of the fP + flND fraction obtained by milling of the fluorescent microdiamond (shown in figure 7 (c)) and purification of the resulting fMD fraction. (c) AFM dimensions of a 10 nm bright fND diamond nanocrystal marked with a square frame in panels (a) and (b). (d) Photoluminescence spectrum of the same nanodiamond,

corresponding to the NV center emission. (e) Second-order fluorescence autocorrelation function for the same nanodiamond. Note that the dip of photon counting statistics curve is related to 12 emitters in the nanocrystal. Analysis of contrast of such a dip allows us to count the number of fluorescing defects.

The brightness of diamond nanocrystals is crucial for their application as ftuorescent markers. For NV-doped nanodiamonds the saturated ftuorescence signal is defined by the number of NV defects. Note that estimation of brightness based on fluorescence intensity signal is not quantitative because the detected signal can be affected by the sensitivity of the experimental set-up. Recently it was shown that exact information on the number of color centers can be obtained by a quantum optical analysis of emitted light [55]. A single NV defect never emits two photons at once because the emission of a photon projects the single quantum system into the ground state (owing to energy conservation). Hence subPoissonian photon statistics, called 'photon antibunching', is found as a dip in the second-order correlation function (for details see the reference) is a signature of a single emitter [61]. When several defects are present in a nanodiamond the probability of detecting two photons is not zero anymore. The contrast of antibunching dips scale as $1 / N$, where $N$ is the number of color centers. Figure $8(e)$ shows the measured autocorrelation function for a selected nanocrystal. The contrast of the antibunching dip is 0.08 , indicating that 12 centers are present in the emission.
Note that, assuming that the nanocrystal is quasi-spherical, its diameter can be measured independently from AFM $z$ coordinates (figure $8(\mathrm{c})$ ), allowing approximation of the concentration of NV defects in nanostructured diamond material.

\section{Conclusions and prospects}

We present here for the first time a high yield method to produce isolated ultrasmall quasi-spherical diamond nanoparticles with dimensions less than or equal to $10 \mathrm{~nm}$. The starting material is a highly crystalline HPHT micronsized diamond and the production has an overall yield of about $15 \%(\mathrm{w} / \mathrm{w})$ of the initial microdiamond for a $24 \mathrm{~h}$ milling time. This yield is several orders of magnitude higher than the present ones [21]. The nanodiamonds obtained here present new features: they are made of c-diamond nanocrystals with controlled size, shape and composition, a very high crystallinity and little impurity but selected doping heteroatoms such as isolated substitutional nitrogen.

U1trasmall ftuorescent nanodiamonds will have outstanding potential applications, for instance in physics or biology. 
Owing to long coherence time even at room temperature, spins associated with single NV defects are expected to be important for use in future nanodevices, such as qubits for quantum computing. NV-doped nanodiamonds can also be used as magnetic field sensors reaching atomic (sub$\mathrm{nm}$ ) spatial resolution and sensitivity of the order of $\mu \mathrm{G}$. Furthermore, availability of small nanocrystals with NV defects located close to the surface of diamond allows their use as sensors for external spins. In biology, the possibility to label molecules (nucleic acids, peptides or proteins) with fluorescent nanodiamonds opens novel prospects for quantitative biology (DNA chip, quantitative PCR, immunodetection of gene products in health and disease) since they sustain long term repetitive measurements which is not achievable with conventional fluorophores. Ultrasmall fluorescent nanodiamonds would also be ideally tailored for single-particle tracking for subcellular dynamics: such a small size reduces dramatically the impact on diffusion in cellular media and the absence of blinking makes trajectory reconstruction much easier, with less need for tracking algorithms to solve the frame-to-frame correspondence problem $[1,62,63]$. Finally, if the nanodiamonds are produced from raw HPHT microcrystals, they should advantageously replace non-fluorescent diamond nanoparticles presently used in numerous applications, such as seeding substrates for thin film CVD diamond synthesis [64, 65], hard disc computer industry [66], bioelectronics [67] or for the next generation of integrated circuits [68].

The observations made in the present work will be useful to adjust at will nanodiamond properties with minimal loss in crystallinity for any of these specific applications and needs (particle size, shape, composition: NV content, nanoparticle doping with selected heteroelements, surface functionalization). The industrial scale production of ultrasmall nanodiamonds represents a breakthrough toward these objectives.

\section{Acknowledgment}

This research was supported by the European Commission with financial support of the project Nano4Drugs under contract LSHC-CT-2005-019102.

\section{References}

[1] Balasubramanian G et al 2008 Nanoscale imaging magnetometry with diamond spins under ambient conditions Nature 455 648-51

[2] Barnard A S and Sternberg M 2005 Substitutional nitrogen in nanodiamond and bucky-diamond particles J. Phys. Chem. B 109 17107-12

[3] Chang Y C et al 2008 Mass production and dynamic imaging of fluorescent nanodiamonds Nat. Nanotechnol. $3284-8$

[4] Hartl A, Schmich E, Garrido J A, Hernando J, Catharino S C R, Walter S, Feulner P, Kromka A, Steinmuller D and Stutzmann M 2004 Protein-modified nanocrystalline diamond thin films for biosensor applications Nat. Mater. 3 $736-42$

[5] Wu E, Jacques V, Zeng $H$, Grangier $P$, Treussart $F$ and Roch J-F 2006 Narrow-band single-photon emission in the near infrared for quantum key distribution Opt. Express 14 1296-303
[6] Greentree A D, Fairchild B A, Hossain F M and Prawer S 2008 Diamond integrated quantum photonics Mater. Today $1122-31$

[7] Wrachtrup J and Jelezko F 2006 Processing quantum information in diamond J. Phys.: Condens. Matter 18 S807-24

[8] Davies G and Collins A T 1993 Vacancy complexes in diamond Diamond Relat. Mater. $280-6$

[9] Gruber A, Dräbenstedt A, Tietz C, Fleury L, Wrachtrup J and Borczyskowski C V 1997 Scanning confocal optical microscopy and magnetic resonance on single defect centres Science 276 2012-4

[10] Rabeau J R, Reichart P, Tamanyan G, Jamieson D N, Prawer S, Jelezko F, Gaebel T, Popa I, Domhan M and Wrachtrup J 2006 Implantation of labelled single nitrogen vacancy centers in diamond using $15 \mathrm{~N}$ Appl. Phys. Lett. 88023113

[11] Schrand A M, Huang H, Carlson C, Schlager J J, Osawa E, Hussain S M and Dai L 2007 Are diamond nanoparticles cytotoxic? J. Phys. Chem. B $1112-7$

[12] Zaitsev A 2001 Optical Properties of Diamond: A Data Handbook (Berlin: Springer)

[13] Yang W et al 2002 DNA-modified nanocrystalline diamond thin-films as stable, biologically active substrates $N a t$. Mater. 1253-7

[14] Osswald S, Havel M, Mochalin V, Yushin G and Gogotsi Y 2008 Increase of nanodiamond crystal size by selective oxidation Diamond Relat. Mater. 17 1122-6

[15] Iakoubovskii K, Mitsuishi A and Furuya A 2008 High-resolution electron microscopy of detonation nanodiamond J. Nanotechnol. 108155705

[16] Hu S, Tian F, Bai P, Cao S, Sun J and Yang J 2009 Synthesis and luminescence of nanodiamonds from carbon black Mater. Sci. Eng. B $15711-4$

[17] Ting C C, Young T-F and Jwo C-S 2007 Fabrication of diamond nanopowder using microwave plasma torch technique Int. J. Adv. Manuf. Technol. 34 316-22

[18] Stacey A, Aharonovich I, Prawer S and Butler J E 2009 Controlled synthesis of high quality micro/nano-diamonds by microwave plasma chemical vapor deposition Diamond Relat. Mater. 18 51-5

[19] Kennedy T A, Colton J S, Butler J E, Linares R C and Doering P J 2003 Long coherence times at $300 \mathrm{~K}$ for nitrogen-vacancy center spins in diamond grown by chemical vapor deposition Appl. Phys. Lett. 83 4190-2

[20] Kalish R, Uzan-Saguy C, Philosoph B, Richter V, Lagrange J P, Gheeraert E, Deneuville A and Collins A T 1997 Nitrogen doping of diamond by ion implantation Diamond Relat. Mater. 6 516-20

[21] Morita Y, Takimoto T, Yamanaka H, Kumekawa K, Morino S, Aonuma S, Kimura T and Komatsu N 2008 A facile and scalable process for size-controllable separation of nanodiamond particles as small as $4 \mathrm{~nm}$ Small 4 2154-7

[22] Beveratos A, Brouri R, Gacoin T, Poizat J P and Grangier P 2001 Nonclassical radiation from diamond nanocrystals Phys. Rev. A 64061802

[23] Faklaris O, Garrot D, Joshi V, Druon F, Boudou J, Sauvage T, Georges P, Curmi P and Treussart F 2008 Detection of single photoluminescent diamond nanoparticles in cells and study of the internalization pathway Small 4 2236-9

[24] Sonnefraud Y, Cuche A, Faklaris O, Boudou J P, Sauvage T, Roch J F, Treussart F and Huant S 2008 Diamond nanocrystals hosting single nitrogen-vacancy color centers sorted by photon-correlation near-field microscopy Opt. Lett. 33 611-3

[25] Tisler $\mathbf{J}$ et al 2009 Fluorescence and spin properties of defects in single digit nanodiamonds $N$ ano Lett. submitted

[26] Boudou J P and Curmi P A 2008 Method to produce light-emitting nano-particles of diamond EP1990313 (A1) (ed INSERM) 
[27] Lawson S, Davies G, Collins A T and Mainwood A 1992 Migration energy of the neutral vacancy in diamond J. Phys.: Condens. Matter 4 L125-31

[28] Iakoubovskii K, Kiflawi I, Johnston K, Collins A, Davies G and Stesmans A 2003 Annealing of vacancies and interstitials in diamond Physica B 340-342 67-75

[29] Gaffet $\mathrm{E} 1991$ Ball milling: an $E-\mathrm{v}-T$ parameter phase diagram Mater. Sci. Eng. A $135291-3$

[30] Gaffet E, Bernard F, Niepce J-C, Charlot F, Gras C, Caër G L, Guichard J-L, Delcroix P, Mocellin A and Tillement O 1999 Some recent developments in mechanical activation and mechanosynthesis J. Mater. Chem. 9 305-14

[31] Ungár T, Borbély A, Goren-Muginstein G R, Berger S and Rosen A R 1999 Particle-size, size distribution and dislocations in nanocrystalline tungsten-carbide Nanostruct. Mater. 11 103-13

[32] Maeta H, Matsumoto N, Haruna K, Saotome T, Ono F, Sugai H, Ohtsuka H and Ohashi K 2006 The characterization of synthetic and natural single crystal diamonds by x-ray diffraction Physica B 376/377 283-7

[33] Patters on A L 1939 The Scherrer formula for x-ray particle size determination Phys. Rev. 56 978-82

[34] Loh K P, Xie X N, Lim Y H, Teo E J, Zheng J C and Ando $\mathrm{T} 2002$ Surface oxygenation studies on 10 0 )-oriented diamond using an atom beam source and local anodic oxidation Surf. Sci. 505 93-114

[35] Ando T, Yamamoto K, Matsuzawa M, Takamatsu Y, Kawasaki S, Okino F, Touhara H, Kamo M and Sato Y 1996 Direct interaction of elemental fluorine with diamond surfaces Diamond Relat. Mater. 5 1021-5

[36] Hird J R and Field J E 2004 Diamond polishing Proc. R. Soc. A $4603547-68$

[37] Zhao D S, Zhao M and Jiang Q 2002 Size and temperature dependence of nanodiamond-nanographite transition related with surface stress Diamond Relat. Mater. 11 234-6

[38] Barnard A S 2006 Theory and modeling of nanocarbon phase stability Diamond Relat. Mater. 15 285-91

[39] Gaffet E and Harmelin M 1990 Crystal-amorphous phase transition induced by ball-milling in silicon J. Less-Common Met. 157 201-22

[40] Huang J Y 1999 HRTEM and EELS studies of defects structure and amorphous-like graphite induced by ball-milling Acta Mater. 47 1801-8

[41] Shen T D, Zhang J and Zhao Y 2008 What is the theoretical density of a nanocrystalline material? Acta Mater. $563663-71$

[42] Tolkowsky M 1920 Research on the abrading, grinding or polishing of diamond Guilds College, University of London, London

[43] Telling R H, Pickard C J, Payne M C and Field J E 2000 Theoretical strength and cleavage of diamond Phys. Rev. Lett. 845160

[44] Remediakis I N, Kopidakis G and Kelires P C 2008 Softening of ultra-nanocrystalline diamond at low grain sizes Acta Mater. $565340-4$

[45] Gaffet E 1991 Phase transition induced by ball milling in germanium Mater. Sci. Eng. A $136161-9$

[46] Maurice D and Courtney H 1990 The physics of mechanical alloying: a first report Metall. Mater. Trans. A 21 289-303

[47] Chattopadhyay P P, Manna I, Talapatra S and Pabi S K 2001 A mathematical analysis of milling mechanics in a planetary ball mill Mater. Chem. Phys. 68 85-94
[48] Gogotsi Y G, Kailer A and Nickel K G 1999 Materials: transformation of diamond to graphite Nature 401 663-4

[49] Chacham $\mathbf{H}$ and Kleinman L 2000 Instabilities in diamond under high shear stress Phys. Rev. Lett. 854904

[50] van Bouwelen F M, Field J E and Brown L M 2003 Electron microscopy analysis of debris produced during diamond polishing Phil. Mag. 83 839-55

[51] Gubarevich A, Usuba S, Kakudate Y, Tanaka A and Odawara O 2004 Diamond powders less than $100 \mathrm{~nm}$ in diameter as effective solid lubricants in vacuum Japan. J. Appl. Phys. 43 L920-3

[52] Burns R C, Cvetkovic V, Dodge C N, Evans D J F, Rooney M-L T, Spear P M and Welbourn C M 1990 Growth-sector dependence of optical features in large synthetic diamonds J. Cryst. Growth 104 257-79

[53] Collins A T 1980 Vacancy enhanced aggregation of nitrogen in diamond J. Phys. C: Solid State Phys. 13 2641-50

[54] Collins A T, Kanda H and Kitawaki H 2000 Colour changes produced in natural brown diamonds by high-pressure, high-temperature treatment Diamond Relat. Mater. 9113-22

[55] Kanda H, Akaishi M and Yamaoka S 1999 Synthesis of diamond with the highest nitrogen concentration Diamond Relat. Mater. 8 1441-3

[56] Borzdov Y, Pal'yanov Y, Kupriyanov I, Gusev V, Khokhryakov A, Sokol A and Efremov A 2002 HPHT synthesis of diamond with high nitrogen content from an $\mathrm{Fe}_{3} \mathrm{~N}-\mathrm{C}$ system Diamond Relat. Mater. 11 1863-70

[57] Yu R Z, Ma H A, Liang Z Z, Liu W Q, Zheng Y J and Jia X 2008 HPHT synthesis of diamond with high concentration nitrogen using powder catalyst with additive $\mathrm{Ba}\left(\mathrm{N}_{3}\right)_{2}$ Diamond Relat. Mater. 17 180-4

[58] Barnard A S and Sternberg M 2007 Mapping the location and configuration of nitrogen in diamond nanoparticles Nanotechnology 18025702

[59] Tamarat $P$ et al 2008 Spin-flip and spin-conserving optical transitions of the nitrogen-vacancy centre in diamond $\mathrm{New} J$. Phys. 10045004

[60] Jelezko F, Tietz C, Gruber A, Popa I, Nizovtsev A, Kilin S and Wrachtrup J 2001 Spectroscopy of single N-V centers in diamond Single Mol. 2 255-60

[61] Neugart F, Zappe A, Jelezko F, Tietz C, Boudou J P, Krueger A and Wrachtrup J 2007 Dynamics of diamond nanoparticles in solution and cells Nano Lett. 7 3588-91

[62] Maze J R et al 2008 Nanoscale magnetic sensing with an individual electronic spin in diamond Nature $\mathbf{4 5 5}$ 644-7

[63] Saxton M 2008 Single-particle tracking: connecting the dots Nat. Methods 5 671-2

[64] Arnault J C, Saada S, Nesladek M, Williams O A, Haenen K, Bergonzo P and Osawa E 2008 Diamond nanoseeding on silicon: stability under $\mathrm{H}_{2}$ MPCVD exposures and early stages of growth Diamond Relat. Mater. 17 1143-9

[65] Williams O A, Nesladek M, Daenen M, Michaelson S, Hoffman A, Osawa E, Haenen K and Jackman R B 2008 Growth, electronic properties and applications of nanodiamond Diamond Relat. Mater. 17 1080-8

[66] Kurmashev V, Timoshkov Y, Orehovskaja T and Timoshkov V 2004 Nanodiamonds in magnetic recording system technologies Phys. Solid State 46 696-702

[67] Nebel C E, Shin D, Rezek B, Tokuda N, Uetsuka H and Watanabe H 2007 Diamond and biology J. R. Soc. Interface 4 439-61

[68] May P W 2008 The new diamond age? Science 319 1490-1 